\title{
New Immersive Media to Broaden Attention and Awareness
}

\author{
Tony Langford \\ Fovolab \\ Cardiff Metropolitan \\ University, UK \\ tolangford@cardiffmet.ac.uk
}

\author{
Alistair Burleigh \\ Fovolab \\ Cardiff Metropolitan \\ University, UK \\ aburleigh@cardiffmet.ac.uk
}

\author{
Nicole Ruta \\ Fovolab \\ Cardiff Metropolitan \\ University, UK \\ niruta@cardiffmet.ac.uk
}

\author{
Robert Pepperell \\ Fovolab \\ Cardiff Metropolitan \\ University, UK \\ rpepperell@cardiffmet.ac.uk
}

\begin{abstract}
We present a new and immersive form of imaging technology, Fovography, which has shown measurable benefits in the broadening of attention. Narrow fixation on screens, such as tablets and smartphones, and resultant neglect of peripheral visual field may be harmful to visual perception and cognitive functioning, with consequent effects on psychological health and wellbeing. Many immersive technologies and virtual reality systems, which narrow the focus of attention to the central visual area, are used in clinics, surgical theatres, treatments and training, and may be doing harm as well as good. We aim to improve existing imaging technologies, which are based on linear perspective, by developing more perceptually natural technologies that are modelled on human perception and awareness, and incorporate peripheral sensory information for improved naturalistic perspective. We have empirically tested the benefits of Fovography technology in the areas of attention modulation, user preference, immersion, empathy, visual comfort and spatial presence. Potential applications include tools for mindfulness training, spatial awareness, perceptual psychology, and other medical or therapeutic uses. The benefits of our approach include greater reported wellbeing due to decreased anxiety and fixation.
\end{abstract}

Perception. Attention. Awareness. Immersion. Peripheral vision. Imaging. Visualisation. Interdisciplinary.

\section{PERIPHERAL VISION AND ATTENTION}

Normal human vision uses two mobile eyes with a combined angle of view of some 180 o horizontally and 1300 vertically to create a visual field that is approximately oval in shape (Gibson 1950). We see clearly only in the centre of the visual field (the foveal region) while the majority of the visual field is relatively indistinct (the peripheral region) (Anstis 1998).

Natural scene perception and active vision are dynamic, embodied processes that involve the orienting to and tracking of objects that move in and out of our field of view via eye, head and torso rotations (Diaz, Cooper \& Hayhoe 2013). In such natural scene perception, our visual periphery is likely to be much more important than currently recognised by screen-based vision science (Land, Mennie \& Rusted 1999, Loschky et al. 2017)

The peripheral visual field also contains important information for effective cognitive functioning. The area of the brain specialised for detecting objects in peripheral vision, the prostriata, has a direct link to other areas of the brain related to emotions and attention (Hsin-Hao et al. 2012). The prostriata is one of the first areas affected in Alzheimer's disease and associated spatial disorientation and tunnel vision. Visual motion processing is impaired by distinct mechanisms in aging and the transition to Alzheimer's (Kavcic, Vaugh \& Duffy 2011).

Many contemporary media devices, such as smart phones, grab the focus of attention to the foveal area of vision, at the exclusion of the wider peripheral field that is critical to natural visual perception (Lutz, Ranzini \& Meckel 2014). The narrow focus of attention demanded by these devices may be harmful to cognitive functioning, emotion regulation and visual perception ( $\mathrm{Lim}$ et al. 2017, Wilmer, Sherman \& Chein 2017). These technologies are arguably diminishing our natural capacity for rich visual experience.

While scientific literature on the effects of contemporary media devices on cognitive functioning and visual perception is still in its nascent stages, the link between vision and the effects of technology is certainly not new. Frederick Franck (1973) commented in 'The Zen of Seeing' that: '[w]e do a lot of looking: we look through 
lenses, telescopes, television tubes...Our looking is perfected everyday - but we see less and less'.

Les Fehmi (2007) suggests that our predominant attention style is 'narrow objective' - a tense, constricted, survival mode of attention that holds us in a state of chronic stress, and which lies at the root of common psychological conditions including anxiety, depression and Attention Deficit Disorder. And the relationship between peripheral vision and stress-related conditions can go in either direction 'reduced peripheral awareness can increase stress just as increased stress can reduce peripheral awareness' (Gallop 1996). Fehmi advocates that we return to a more relaxed, diffuse, and creative form of attention, 'Open Focus", which lends from four attention styles: narrow, objective, diffuse and immerse.

Narrow objective attention styles are primarily associated with the left hemisphere of the brain, which is analytical and detail-oriented, where the right hemisphere is relational and has greater breadth, flexibility and generosity. lain McGilchrist (2012) illustrates the tension between these two world-views, and argues that the left hemisphere is increasingly taking precedence in the modern world, with cultural conditions that include information overload and increasing amounts of screen time, and negative consequences for health and wellbeing. While with the right hemisphere in the lead, the parasympathetic nervous system activates, opening to social engagement and connection (Porges 2009).

Open Focus is a method to reduce stress and enhance wellbeing by using principles developed in the field of neurofeedback (McKnight \& Fehmi 2008), and is also used by athletes and performers to train for peak performance. By encouraging awareness of the wide array of sensory experiences - and the space between those experiences - synchronous alpha brainwaves generate a relaxed alertness and effortless attention that results in an ease and flow of experience. This is accomplished by invoking attention to space, peripheral vision and a broadening of awareness.

\section{FOVOGRAPHY}

Fovography naturalistically emulates visual space rather than merely simulating light rays and is based on the structure of human visual perception rather than the geometry of optics. It offers a more naturalistic form of imaging than is provided by conventional cameras and computer graphics systems which universally rely on linear perspective, for a long time seen by artists as ineffective in representing the full scope of human vision.
Evidence from cognitive and perceptual psychology studies shows that media formats based on linearperspective driven screens can conflict with naturally evolved patterns of human visual behaviour (Tatler et al. 2011, Smith 2012). Most scientific understanding of how we process visual scenes is based on using static, 2D photographs presented on screens as a proxy for the real world. The presence of the screen edge and the inability of the viewer to influence the viewpoint depicted significantly constrains the behaviours and cognitive processes of viewers. By relying on framed linear perspective images, our current scientific insight into natural visual cognition may be inherently flawed (Mital et al. 2011, Tatler 2007).

The Fovography technology derives from six years of multidisciplinary research, drawing on artistic practice such as painting and drawing, the history of art, the philosophy of perception, the psychology of visual perception, and computer graphics (Pepperell 2012, Pepperell 2017, Pepperell \& Burleigh 2014). Until very recently it was not feasible to implement these systems in working image technology due to their geometric complexity. The artistic perceptual mapping process is now implemented as a general-purpose computer graphics pipeline running in shader code on graphics processing units. It can manipulate any $2 \mathrm{D}$ or $3 \mathrm{D}$ image source to produce perceptually natural projections on any display. It is a form of natural media (Smith et al. 2017) that can overcome many of the problems associated with imaging technologies based on linear perspective projection. For example, it can:

- Depict a wide field of view (>180 degrees) with minimal apparent distortion.

- Capture and display binocular data without head-mounted displays or 3D glasses.

- Differentiate between the central and peripheral visual fields.

- Respond dynamically to user behaviour in terms of eye, head and body motion.

- Present a perceptually naturalistic firstperson point of view.

We are addressing a long-standing debate about the nature of human visual experience and how it is depicted (Fresnoy 1695, Kemp 1990). Figures like Ernst Gombrich and James Gibson argued that linear perspective images accurately simulate the light rays emanating from a scene and therefore the scene itself (Gibson 1971). Numerous artists have disputed this, including more recently David Hockney, arguing that linear perspective narrows visual experience, lacks depth, and is unresponsive to the dynamics of active vision. Images based on linear perspective may be grossly constraining the active viewer's perceptual abilities and limiting the detection of peripheral features. In response, artists 
have developed alternative systems of depiction for more effectively conveying our experience of space, including curvilinear and non-linear forms of pictorial perspective, which they claim more faithfully represent 'how' rather than 'what' we see (Gombrich 1972).

\section{FOVOGRAPHY AND MINDFULNESS}

Fovography replicates the non-duality of human visual perception, in a way that allows us to see what is actually there, in the present moment (Dunne 2011). Subject (seer) and object (what is seen) unite in the moment of seeing, as opposed to the separation that occurs with the more constrained act of looking.

Pre-conceived ideas of the mind and ingrained or conditioned thinking can often obscure our capacity to 'see' clearly. Through the practice of mindfulness we can release attachment to thoughts by bringing awareness to the breadth of sensory experience in the present moment. Jon Kabat-Zinn (2005) has called this awareness an orthogonal reality because it is 'akin to the transition from a twodimensional flatland into a third more spacious dimension, orthogonal to the other two'. With this broader and more spacious perspective, new possibilities for freedom, acceptance, creativity and compassion emerge.

This shift of focus happens as an internal process around mental processing and bodily sensations, through the mind as an embodied and relational process (Siegel 2009). So our research question is, can we influence this attentional shift using an external visual source? Can we show how Fovography can manipulate the focus of attention and broaden awareness?

We hypothesized that an artistic representation of the human visual field would help in broadening the attention and awareness of technology users. We predicted that representing the periphery of a scene on a computer screen would counterbalance the 'narrow objective' form of attention that is associated with stress and anxiety. We empirically tested whether Fovography technology provided any benefits over existing imaging technologies in the following key areas:

- Modulation of attention by image properties.

- Mindfulness training with attention direction.

- Reported levels of user preference, immersion, visual comfort, and spatial presence.

Our hypothesis was that the human visual system prefers image geometries that mimic the natural structure of visual space to those that do not. In particular, we investigated whether including information from the peripheral visual field that is normally ignored by conventional imaging devices affects psychological states such as attention, mindful awareness, and sense of presence.

\section{MODULATION OF ATTENTION}

We employed the well-established Posner Cueing Task (Posner 1980) using a custom-built gamified apparatus to test whether altering the geometrical structure of the image space affected participants' visual attention (Ruta et al. 2017). We hypothesised that one of the key features of Fovography is that it enhances the area of fixed attention within an image, drawing and holding the viewer's attention more strongly than in conventional linear perspective images.

Each participant performed the attention computerbased task comparing three different versions of the same image: Fovography, Fisheye and Linear Perspective (LP). We randomly assigned participants to two different groups: an experimental and control condition, with 10 participants per group. In the first one we presented the target in the geometrically correct position, changing its size and location according to the projection that was presented; in the second one we presented the target always in the same position and at the same size, while the background changed accordingly to the geometry. This allowed us to test if the image geometry directly influenced the participant's voluntary shift of attention to a cued location. Overall results showed an advantage - faster reaction times (RTs) - if the target was preceded by the cue. Moreover there was a significant difference between the three geometries, mainly due to participants being faster if the task was performed having a LP image as a background. This might be due to participants being more familiar to LP images, but also the cue size being relatively bigger in the LP image because of its stretched periphery compared to Fovo and Fisheye. Participants were significantly slower shifting their attention away from the central area to the peripheral target if they were looking at a Fovo or a Fisheye image compared to LP, meaning that Fovo artistic manipulation and curved geometry fixed more participants' attention to the centre of the image compared to LP.

Overall, the data reported a significant difference between the two groups, with participants significantly faster in the control condition compared to the experimental one. However in the control condition the advantage for the cued location was not significant, suggesting that our manipulation successfully influenced the voluntary shift of attention. 
The Fovography geometry slowed participants' reaction times compared to the linear perspective condition, which is consistent with our hypothesis. This allows us to conclude that altering the geometry of a given scene can affect the distribution of attention across the visual field. This could affect the way we design and use imaging technologies in a variety of contexts, not least diagnostic, clinical and therapeutic.

\section{MINDFULNESS TRAINING TOOL}

To evaluate whether Fovography technology could have beneficial effects on mindfulness training we devised a prototype mindfulness training tool. In designing the tool we drew on the Phenomenological Matrix of Mindfulness-Related Practices proposed by Antoine Lutz et al. (2015), which identifies four qualitative dimensions characterizing mindfulness practice: aperture, clarity, stability and effort. We tested if our technology could manipulate these four dimensions of attention by altering image properties and therefore showing how it is possible to shift certain mind states of constricted or narrow attention, such as addictive craving, mind-wandering or rumination (Lutz et al. 2015).

In mindfulness, attention is focused on either a single point such as the breath or a bodily sensation (Focused Attention), or a wider focal point such as a combination of sensations (Open Monitoring), but the aim is to notice and observe when the mind wanders, and each time that happens to gently bring it back to the intended area of attention (Hasenkamp et al. 2011).

Our Mindfulness-training tool consisted of two stages: the first one manipulated the parameters of stability and effort, illustrating the 'Focused Attention' style of mindfulness practice. The second one added clarity and aperture, illustrating the 'Open Monitoring' style. Both stages began with a moving visual target: the longer the participant fixed their attention on it, the steadier it became, moving to the centre of the screen. During the first stage, participants were instructed to focus on a floating red ball, using the visual to anchor them in the present moment, while also focusing on their breath and body sensations. A psychologist read a fixed protocol guiding the participant during the training. The session started with an $8 \mathrm{~cm}$ ball whose size was reduced or increased during the session, according to the participant's ability to focus on it. This allowed us to manipulate their perceived effort during the experiment.

During the second stage a floating image of a candle appeared on the screen. As the participant focused on it, the image became wider, gradually revealing a first-person perspective view of the candle (see Figure 1). The aim of this stage was to induce a state of 'Open Monitoring', asking participants to broaden their attention to the whole scene on the screen, and then the borders of the screen itself and then the furniture and sounds of the room around them, without shifting the gaze of their eyes.

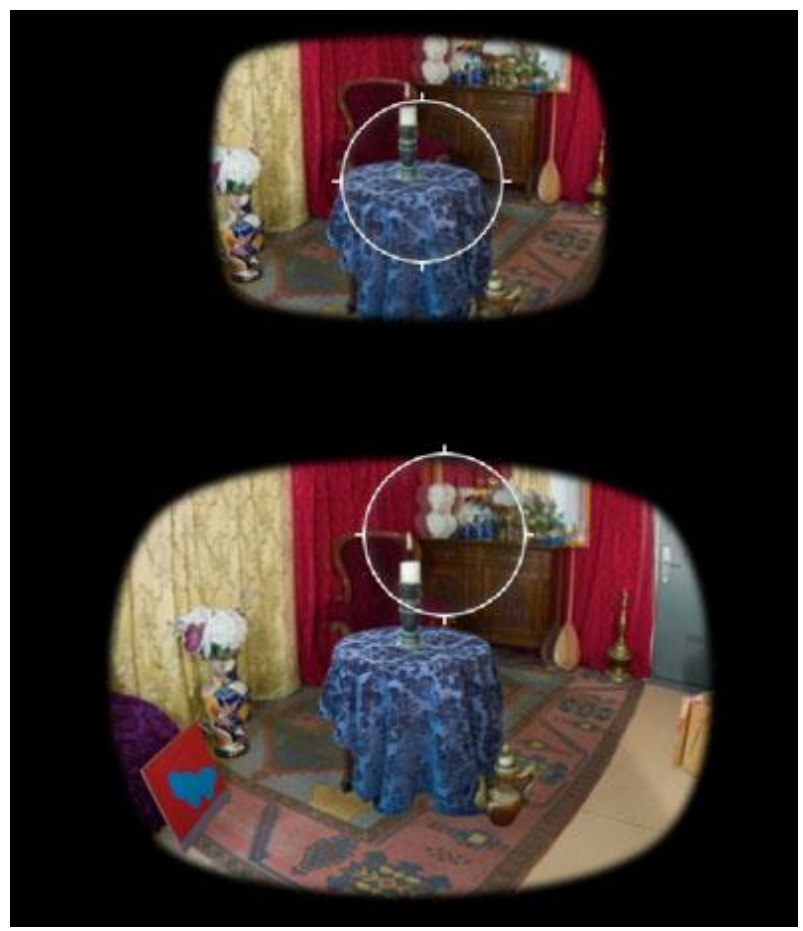

Figure 1: The image of the candle becomes wider as the participant focuses on it.

We developed a customized version of the Intrinsic Motivation Inventory (Markland \& Hardy 1997) to collect participants' ratings on their perceived value for both the visuals and the spoken protocol. We also collected participants' ratings on perceived manipulation of the dimensions of attention from Lutz's Phenomenological Matrix.

Participants rated their agreement on a series of statements on a 7-point Likert scale, where 1 was "not at all" and 7 was "very much". Results showed that participants rated both the visuals and the spoken protocol valuable (visuals average rating= 4.71; speech average rating $=5.52$ ). During the first session, participants perceived that the visuals directly manipulated their effort. Results showed that increasing of the ball size made the focused attention task easier (average $=5.42$ ). During the second session, most participants found that the widening of the image was helpful in directly helping to broaden their attention (Mode=6) and that the candle was directly manipulating the clarity of their perception (Mode=4).

Results suggest that the mindfulness training tool directly manipulates key dimensions of mindfulness 
experience, giving novice meditators visual feedback on their attention. We showed an improvement of the meditation experience, increasing the participants' perception of awareness and providing a more spacious perspective on their mental state. The tool was able to manipulate the dimensions of attention in a beneficial way, and was rated by participants as helpful in achieving a more mindful state.

\section{IMAGE EVALUATION AND COMPARISON WITH OTHER IMMERSIVE SYSTEMS}

In comparisons between a real three-dimensional space and three different screen formats conventional linear perspective, a fisheye lens, and Fovography - participants rated the Fovography representation significantly higher compared to the others in terms of preference, comfort, spatial presence and ecological validity.

We then investigated whether a Fovo synthetic vision engine (SVE) could produce comparable levels of immersion and spatial presence to other widely used immersive technologies: virtual reality and 180 degree projections. The SVE is an interactive image that modifies the geometry of a scene according to where the participant is looking using eye tracking hardware. It employs the principles of Fovography to create a more immersive and naturalistic image of a space than can be achieved with conventional linear perspectival methods.

We asked participants to rate their experience of interacting with a 3D environment in four different conditions: the Fovo SVE, a conventional linear perspective projection on a computer screen, a 180-degree projection PEL screen (see Figure 2), and a virtual reality headset. The results showed that Fovography had similar levels of spatial presence and ecological validity to the $180^{\circ}$ projection, and was not significantly different from VR for ecological validity. Fovography was comparable to the projection on ratings for immersion, and the linear perspective projection was rated least favourite on all measures. The results suggest that Fovography, which requires no special glasses or headset, or expensive screens, can deliver levels of immersion and liking comparable to those achieved with more traditional immersive systems. This could have beneficial consequences for the design and use of imaging systems in a range of applications.

The isolation / confinement / immersion of virtual reality can be seen as an advantage when the desired objective is to expose people to a specific situation or phobia (Valmaggia et al. 2016). Virtual reality immersive technologies have also been shown to be effective as a tool for relaxation and in the treatment of chronic pain (Nazemi, Gromala \& Karamnejad 2014). Virtual reality can however be unsuitable for some users who may be overwhelmed by the isolation and immersion into another perspective (Madary 2016).

The near-eye displays in VR and AR headsets, which are increasingly used in training, therapy and intervention, are inherently uncomfortable due to the extreme demands they make on the accommodation muscles of the eyes, a problem that becomes greater as users age (Konrad et al. 2017, Zhang 2003). Head-mounted displays entail multiple problems and costs, and training systems are often limited to one user at a time (Rolland \& Fuchs 2000). They also have an unnaturally narrow field of view that encourages excessive head movements by way of compensation. Furthermore, the viewer's body is not included within the media content, which can promote disorientation as well as a sense of separation rather than connection to the viewer.

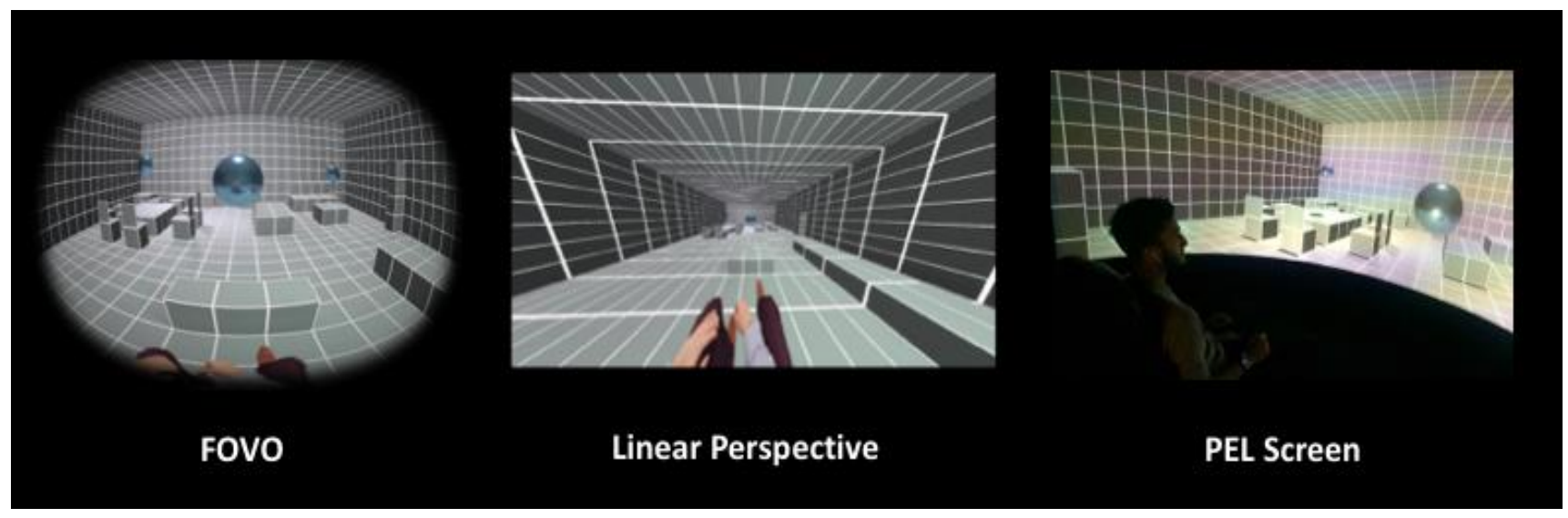

Figure 2: Participants compared the same content in these three technologies in addition to a VR headset. 
In such cases a sense of shared observation, as opposed to immersion, may be more effective, and is also more aligned with mindfulness, where empathy is balanced with equanimity, selfcompassion and the shared immersion of social engagement. Virtual reality and other more conventionally immersive media on computer or mobile device screens do not allow this broader perspective and sense of objectivity, as the user's attention is too narrowly focused or constrained.

\section{FUTURE APPLICATIONS}

Fovography therefore has potential as a visual technology to support mindfulness training that encourages greater self-awareness and broadening of attention, and may be able to supplement or support the work of scarce qualified personnel. There is a current deficit of mindfulness trainers, which is preventing wider uptake of the practice, with poor adherence rates after training due to lack of follow up (Bermingham 2015).

A further application will be to expand our understanding of the flexibility/change in the perceptual parameters of the visual field, such as focus / aperture of attention, that may occur with long-term mindfulness practice. This would facilitate the effectiveness of mindfulness training to provide an objective outcome measure of mindfulness-based interventions.

The effective manipulation of central and peripheral visual information in images may also improve legibility in visual media for patients with visual impairments such as macular degeneration and glaucoma, and amblyopia where stereoscopic depth perception is impaired.

Fovography also has the potential to bring a broader awareness to body sensations as well as the 'felt sense' (Gendlin 1992) of the embodied mind (Rosch, Thompson \& Varela 1991). Enhanced presence / agency / embodiment from mindfulness training can increase acceptance / trust of interoceptive body signals (Farb et al. 2015). Applications using natural media could therefore have a clear benefit to therapies such as Somatic Experiencing (Payne, Levine \& CraneGodreau 2015), a method aimed at relieving mental and physical trauma-related health problems by focusing on the client's perceived body sensations.

Eye Movement Desensitisation and Reprocessing (EMDR) is another therapy commonly used for the treatment of trauma-related symptoms, and has integrated eye movements into a method for resolving distressing content in the brain (Lee \& Cuijpers 2013). This concept could be developed further using Fovography techniques that broaden awareness to a depth of seeing that results in decreased fixation and anxiety, and greater 'flow' and wellbeing. There is further scope for applying the principles of Fovography to increase our understanding of flow states and peak performance, as well as how neurodiverse populations perceive visual space and spatial awareness, and how devices might be designed that improve current diagnosis, monitoring, and treatments.

\section{CONCLUSIONS}

In summary, the experiments conducted are encouraging for the approach we are taking to creating new and immersive forms of imaging technology. Collectively our results suggest that a method of depicting visual space based on the research of artists can have multiple beneficial effects in a range of contexts.

We have shown that by modifying linear perspective using the spatial geometry derived from human perception, we can affect the distribution of attention across the visual field, and that Fovography is able to manipulate and broaden attention in a beneficial way, in addition to delivering higher ratings of liking and immersion comparable to those achieved with more traditional immersive systems, and greater reported wellbeing due to decreased anxiety and fixation.

Perceptually natural immersive technologies have the potential to engender a sense of empathy and connection with another's perspective as well as a broader awareness of the viewer's own point of view. This 'seeing yourself seeing' can be compared to the noticing and acceptance of thoughts, sounds and sensations in mindfulness practice, all of which promotes 'executive control' in the brain (Teper \& Inzlicht 2012). An appreciation of the breadth of visual experience, including the periphery, can be compared to the noticing of the hidden beauty within the ordinary, or mundane, and how in mindfulness practice fresh and alert attention is paid to each incoming stimulus, 'no matter its valence or familiarity' (Antonova, Chadwick \& Kumari 2015).

Fovography may offer a more efficacious alternative to virtual and augmented reality systems currently being used to promote mindfulness and wellbeing, and has the potential to enable a redirection or diffusion of attention to a more open, connected and relational state of presence and awareness.

Further research is necessary to develop perceptually natural technologies that incorporate or combine other aspects of sensory experience, 
including auditory, proprioceptive and interoceptive. Paired with psychophysiological measures such as EEG brainwave activity and heart rate variability this could provide further multiple applications with medical and therapeutic uses.

\section{ACKNOWLEDGEMENTS}

The authors acknowledge the funding received from a Wellcome Seed Award in support of the experiments and trials detailed in this paper.

\section{REFERENCES}

Antonova, E., Chadwick, P., and Kumari, V. (2015) More Meditation, Less Habituation? The Effect of Mindfulness Practice on the Acoustic Startle Reflex, Plos One, 10.5.

Anstis, S. (1998) Picturing peripheral acuity. Perception, 27:817-825.

Bermingham, K. (2015). Mindfulness: an effective mental health treatment but not a panacea. The Guardian. http://bit.ly/2G3u6Qr (retrieved 12 September 2017).

Diaz, G., Cooper, J. and Hayhoe, M. (2013) Memory and prediction in natural gaze control. Philosophical Transactions of the Royal Society of London Biological Sciences, 368(1628):20130064.

Dunne, J. (2011) Toward an Understanding of Nondual Mindfulness, Contemporary Buddhism 12(1):71-88.

Farb, N., Daubenmier, J., Price C. J., Gard, T., Kerr, C., Dunn, B. D., Klein, A. C., Paulus M. P, and Mehling, W. E. (2017) Interoception, Contemplative Practice, and Health. Frontiers Research Topics.

Fehmi, L. and Robbins, J. (2008) The open-focus brain: harnessing the power of attention to heal mind and body, Trumpeter, Boston.

Franck, F. (1973) The Zen of Seeing, Wildwood House, London.

Gendlin, E. (1992) The primacy of the body, not the primacy of perception, Man and World, 25(3):341353.

du Fresnoy, C. A. (1695) De arte graphica; or The art of painting. Translated by J. Dryden, J. Heptinstall for W. Rogers, London.

Gallop, S. (1996), Peripheral Visual Awareness: The Central issue. Journal of Behavioural Optometry, 7 (6):151-155.

Gibson, J. J. (1950) The Perception of the Visual World, Houghton Mifflin, Boston.
Gibson, J. J. (1971) The Information Available in Pictures. Leonardo, 4(1):27-35.

Gombrich, E. (1972) The 'What' and the 'How': Perspective Representation and the Phenomenal World. In: Rudner, R. and Scheffler, I. (eds.) (1972) Logic and Art: Essays in Honor of Nelson Goodman. Bobbs Merrill, New York.

Gombrich, E. (1982) The Image and the Eye: Further Studies in the Psychology of Pictorial Representation, Phaidon, London.

Hasenkamp, W., and Barsalou, L. (2012) Effects of Meditation Experience on Functional Connectivity of Distributed Brain Networks. Frontiers in Human Neuroscience, 6.

Hockney, D. (1987) The Way I See It, Thames \& Hudson, London.

Hsin-Hao, Y., Chaplin, T. A., Davies, A. J., Verma, R., and Rosa, M.G.P. (2012) A Specialized Area in Limbic Cortex for Fast Analysis of Peripheral Vision, Current Biology, 22(14):1351-1357.

Kabat-Zinn, J. (2005) Coming to Our Senses, Piatkus Books, London.

Kavcic, V., Vaughn, W., and Duffy, C. J. (2011) Distinct Visual Motion Processing Impairments In Aging and Alzheimer's Disease. Vision Research, 51(3):386-395.

Kemp, M. (1990) The science of art: Optical themes in western art from Brunelleschi to Seurat., CT: Yale University Press, New Haven

Konrad, R., Padmanaban, N., Molner, K., Cooper, E. A. and Wetzstein, G. (2017) Accommodationinvariant computational near-eye displays. ACM Transactions on Graphics, 36(4):88.

Land, M. F., Mennie, N., and Rusted, J. (1999) The roles of vision and eye movements in the control of activities of daily living. Perception, 28:1311-1328.

Lee, C. W., and Cuijpers, P. (2013) A Metaanalysis of the Contribution of Eye Movements in Processing Emotional Memories. Journal of Behavior Therapy and Experimental Psychiatry 44(2):231-239.

Lim, J., Chang, S. H., Lee, J. and Kim, K. (2017) Effects of smartphone texting on the visual perception and dynamic walking stability. Journal of exercise rehabilitation, 13(1):48.

Loschky, L., Nuthmann, A., Fortenbaugh, F., and Levi, D. M. (2017) Scene perception from central to peripheral vision. Journal of Vision, 17(1):1-5.

Lutz, A., Jha, A. P., Dunne, J. D. and Saron, C. D. (2015) Investigating the Phenomenological Matrix of Mindfulness-related Practices from a Neurocognitive Perspective. American Psychologist, 70(7):632-658. 
Lutz, C., Ranzini, G., Meckel, M. (2014) Stress 2.0: Social Media Overload Among Swiss Teenagers. Communication and Information Technologies Annual Studies in Media and Communications, pp.3-24.

Madary, M., and Metzinger, T. K. (2016) Real Virtuality, A Code of Ethical Conduct. Recommendations for Good Scientific Practice and the Consumers of VR-Technology. Frontiers in Robotics and Al, 3, n. pag. Web.

Markland, D., and Hardy, L. (1997) On the factorial and construct validity of the Intrinsic Motivation Inventory: Conceptual and operational concerns. Research Quarterly for Exercise and Sport, 68(1):20-32.

McGilchrist, I. (2009) The Master and His Emissary, Yale University Press, London.

McKnight, J. T. and Fehmi, L. G. (2008) Attention and Neurofeedback Synchrony Training: Clinical Results and Their Significance. Journal of Neurotherapy, 5(1-2):45-61.

Mital, P.K., Smith, T. J., Hill, R. L., and Henderson, J. M. (2011) Clustering of gaze during dynamic scene viewing is predicted by motion. Cognitive Computation, 3(1):5-24.

Nazemi, M., Gromala, D., and Karamnejad, M. (2014), Virtual Reality as Analgesia: An Alternative Approach for Managing Chronic Pain, International Journal of Creative Interfaces and Computer Graphics, 5(2).

Payne, P., Levine, P and Crane-Godreau, M., (2015) Somatic experiencing: using interoception and proprioception as core elements of trauma therapy, Frontiers in Psychology, 6(93).

Pepperell, R. (2012) The perception of art and the science of perception; Pepperell, R.; (2017) Imaging Human Vision: An Artistic Perspective. Human Vision and Electronic Imaging, Proc. of SPIE-IS\&T Electronic Imaging.

Pepperell, R. and Burleigh, A. (2014) Improvements in and relating to image making. International Patent Treaty Cooperation, WO2014122477 A1.

Panofsky, E. (1997) Perspective as Symbolic Form. Translated by C. S. Wood. Zone Books, New York.

Posner, M. I. (1980) Orienting of attention, Quarterly Journal of Experimental Psychology, 32(1):3-25.

Porges, S. (2009) The polyvagal theory: New insights into adaptive reactions of the autonomic nervous system, Cleve Clin J Med., 76(Suppl. 2): S86-S90.
Rolland, J. P. and Fuchs, H. (2000) Optical versus video see-through head-mounted displays in medical visualization. Presence: Teleoperators and Virtual Environments, 9(3):287-309.

Rosch, E., Thompson L., and Varela, F. (1991) The embodied mind, Cognitive Science and Human Experience.

Ruta, N., Burleigh, A., Barratt, E., and Pepperell, R. (2017) How does image geometry affect attention? Developing a novel gamified version of spatial orienting paradigm. Poster presented at the European Conference on Visual Perception 2017, Berlin, Germany.

Siegel, D. J. (2009) Mindful awareness, mindsight, and neural integration. The Humanistic Psychologist, 37(2):137-158.

Smith, C. H., Rasool, J., Lambert, N., Pepperell, R., Langford, T., Korkovelou, L. and Burleigh, A. (2017) Electronic Visualisation and the Arts (EVA 2017). London, UK, 11-13 July 2017, pp.258-263 BCS, London.

Smith, T. J. (2012) The attentional theory of cinematic continuity. Projections, 6(1):1-27.

Tatler, B. W. (2007) The central fixation bias in scene viewing: Selecting an optimal viewing position independently of motor biases and image feature distributions. Journal of Vision, 7(14):4.117.

Tatler, B. W., Hayhoe, M. M., Land, M. F. and Ballard, D. (2011) Eye guidance in natural vision: Reinterpreting salience. Journal of Vision, 11(5).

Teper, R., and Inzlicht, M. (2013) Meditation, mindfulness and executive control: the importance of emotional acceptance and brain-based performance monitoring. Social Cognitive and Affective Neuroscience, 8(1):85-92.

Valmaggia, L. R., Latif, L., Kempton, M. J., and Rus-Calafell, M. (2016) Virtual Reality in the Psychological Treatment for Mental Health Problems: A Systematic Review of Recent Evidence, Psychiatry Research, 236:189-195.

Wilmer, H. H., Sherman, L. E. and Chein, J. M. (2017) Smartphones and Cognition: A review of research exploring the links between mobile technology habits and cognitive functioning. Frontiers in Psychology, 8.

Zhang, S. (2015) The Obscure Neuroscience Problem That's Plaguing VR. Wired. http://bit.ly/2DDLiXn (retrieved 12 September 2017). 